\title{
Association of heart rate trajectories with the risk of adverse outcomes in a community-based cohort in Taiwan
}

\author{
Cheng-Chun Wei ${ }^{1,2}{ }^{,}$Pei-Chun Chen ${ }^{3}$, Hsiu-Ching Hsu ${ }^{4}$, Ta-Chen Su ${ }^{4}$, Hung-Ju Lin ${ }^{4}$, Ming-Fong Chen ${ }^{4,5}$, Yuan- \\ Teh Lee ${ }^{4}$, Kuo-Liong Chien ${ }^{\text {Corresp. } 2,4}$ \\ ${ }^{1}$ Division of Cardiology, Department of Internal Medicine, Shin Kong Wu Ho Su Memorial Hospital, Taipei, Taiwan \\ 2 Institute of Epidemiology \& Preventive Medicine, College of Public School, National Taiwan University, Taipei, Taiwan \\ 3 Department of Public Health, China Medical University, Taichung, Taiwan \\ 4 Department of Internal Medicine, National Taiwan University Hospital, Taipei, Taiwan \\ 5 Cardiovascular Research Laboratory, Cardiovascular Center, Clinical Outcome Research and Training Center, Big Data Center, China Medical University \\ Hospital, Taichung, Taiwan
}

Corresponding Author: Kuo-Liong Chien

Email address: klchien@ntu.edu.tw

Heart rate trajectory patterns integrate information regarding multiple heart rate measurements and their changes with time. Different heart rate patterns may exist in one population, and these are associated with different outcomes. Our study investigated the association of adverse outcomes with heart rate trajectory patterns. This was a prospective cohort study based on the Chin-Shan Community Cardiovascular Cohort in Taiwan. A total of 3,015 Chinese community residents aged $>35$ years were enrolled in a prospective investigation of cardiovascular risk factors and outcomes from 1990 to 2013. The primary outcome was all-cause mortality, and the secondary outcome was a composite of coronary artery disease and cerebrovascular accidents. The following trajectory patterns were identified: stable, $61 \%$; decreased, $5 \%$; mildly increased, $32 \%$; and markedly increased, 2\%. During follow-up (median, 13.9 years), 557 participants died and 217 experienced secondary outcomes. The adjusted hazard ratios of primary and secondary outcomes for participants with a markedly increased trajectory pattern were $1.80(95 \% \mathrm{Cl}, 1.18-2.76)$ and $1.45(95 \% \mathrm{Cl}, 0.67-3.12)$, respectively, compared to those for participants with a stable trajectory pattern. A markedly increased heart rate trajectory pattern may be associated with all-cause mortality risks. Heart rate trajectory patterns demonstrated the utility of repeated heart rate measurements for risk assessment. 


\title{
1 Association of heart rate trajectories with the risk of 2 adverse outcomes in a community-based cohort in \\ 3
}

4

5

6

\author{
Cheng-Chun Wei ${ }^{1,2}$, Pei-Chun Chen ${ }^{3}$, Hsiu-Ching Hsu' ${ }^{4}$, Ta-Chen Su${ }^{4}$, Hung-Ju Lin ${ }^{4}$, Ming- \\ Fong Chen ${ }^{4,5}$, Yuan-Teh Lee ${ }^{4}$, Kuo-Liong Chien ${ }^{1,4}$ \\ ${ }^{1}$ Institute of Epidemiology \& Preventive Medicine, College of Public School, National Taiwan \\ University, Taipei 100, Taiwan \\ ${ }^{2}$ Division of Cardiology, Department of Internal Medicine, Shin Kong Wu Ho Su Memorial \\ Hospital, Taipei, Taiwan \\ ${ }^{3}$ Department of Public Health, China Medical University, Taichung 404, Taiwan \\ ${ }^{4}$ Department of Internal Medicine, National Taiwan University Hospital, Taipei 100, Taiwan \\ ${ }^{5}$ Cardiovascular Research Laboratory, Cardiovascular Center, Clinical Outcome Research and \\ Training Center, Big Data Center, China Medical University Hospital, Taichung 404, Taiwan \\ Corresponding Author: \\ Kuo-Liong Chien \\ No. 17, Xu-Zhou Road, Taipei 100, Taiwan \\ Phone: 886-2-33668017 \\ E-mail:klchien@ntu.edu.tw
}

\begin{abstract}
Heart rate trajectory patterns integrate information regarding multiple heart rate measurements and their changes with time. Different heart rate patterns may exist in one population, and these are associated with different outcomes. Our study investigated the association of adverse outcomes with heart rate trajectory patterns. This was a prospective cohort study based on the Chin-Shan Community Cardiovascular Cohort in Taiwan. A total of 3,015 Chinese community residents aged $>35$ years were enrolled in a prospective investigation of cardiovascular risk factors and outcomes from 1990 to 2013. The primary outcome was all-cause mortality, and the secondary outcome was a composite of coronary artery disease and cerebrovascular accidents. The following trajectory patterns were identified: stable, $61 \%$; decreased, $5 \%$; mildly increased, $32 \%$; and markedly increased, $2 \%$. During follow-up (median, 13.9 years), 557 participants died and 217 experienced secondary outcomes. The adjusted hazard ratios of primary and secondary outcomes for participants with a markedly increased trajectory pattern were 1.80 (95\% CI, 1.18 $2.76)$ and 1.45 (95\% CI, 0.67-3.12), respectively, compared to those for participants with a stable trajectory pattern. A markedly increased heart rate trajectory pattern may be associated with allcause mortality risks. Heart rate trajectory patterns demonstrated the utility of repeated heart rate measurements for risk assessment.
\end{abstract}

Keywords: Heart rate trajectory, resting heart rate, cardiovascular outcomes, all-cause mortality, cerebrovascular accident 


\section{Introduction}

45 Resting heart rate is a straightforward and easily obtainable clinical variable. Epidemiologic expectancies and is associated with an increased risk of adverse outcomes, such as all-cause mortality, cardiovascular diseases, diabetes, cancer, and stroke $[1,2]$. This association has been demonstrated in diverse populations, including healthy individuals [3] and individuals with hypertension [4], established coronary artery disease [5], and heart failure [6]. However, some inconsistencies, such as the role of changes in the heart rate, require further clarification in a longitudinal study.

Most previous studies used a single-point heart rate measurement, such as the baseline heart rate, for analysis [7-9]. The major drawback of this measurement is the possibility of dramatic changes in heart rate between examinations. Therefore, some researchers measured not only baseline heart rates but also differences in heart rates between examinations and found that heart rate differences or changes were more sensitive predictors than a single-point measurement $[4,10,11]$. To study changes in heart rate with outcomes, covariates play an important role. Several covariates, such as age, bodyweight and metabolic factors, vary in the long-term followup and tremendously affect the results. Combining changes in heart rate with time-dependent covariates can help predict the results more accurately [11]. Moreover, different trajectory patterns of changes in heart rate can exist in a population with different outcomes [12].

To the best of our knowledge, few studies have investigated repeated heart rate measurements through heart rate trajectories combined with time-dependent covariates. This study aimed to identify different heart rate trajectory patterns using longitudinal heart rate data and to study the risk of adverse outcomes among different heart rate trajectory patterns.

\section{Materials \& Methods}

\section{Study design and participants}

This prospective cohort study was based on the Chin-Shan Community Cardiovascular Cohort. The details of this cohort have been described previously [13]. All patients provided written informed consent for publication of the information and personal medical history, and the study protocol conformed to the Declaration of Helsinki guidelines. The study was approved by the Institutional Research Board of National Taiwan University Hospital (approval number: 201003001R). Beginning in 1990, a total of 3,602 Chinese community residents aged $>35$ years in Northern Taiwan were enrolled in a prospective investigation of cardiovascular risk factors and outcomes. All-cause mortality and cardiovascular events were monitored until the end of 2013. We excluded participants who were using anti-hypertensive or anti-arrhythmia medications $(n=391)$; whose baseline and follow-up heart rate data were missing $(n=162)$; with key-in error $(n=1)$; whose heart rhythm demonstrated complete atrioventricular block or pacemaker rhythm $(n=5)$; and with atrial fibrillation $(n=28)$. After these exclusions, 3,015 participants were included in the study population. During the 1992-1993 follow-up period, 2,386 participants were available for heart rate analysis. Data were collected from 1,786 and 1,133 participants during the 1994-1995 and 2001-2002 follow-up periods, respectively. The flow diagram of cohort selection is shown in Figure 1. 
89 The participants' anthropometric and lifestyle data, medical history and current health conditions

90 were assessed using interview questionnaires in 2-year cycles through the assistance of trained

91 young faculty and medical students.

92 Definitions of heart rate measurements

93 A 12-lead electrocardiography was performed in each participant to obtain heart rates, and two

94 cardiologists blindly evaluated the results. Participants were instructed to avoid alcohol, food or

95 drink with caffeine, and smoking 8 hours before examination. Four repeated heart rate

96 measurements were obtained during 1990 and 2002. The first heart rate reading was obtained

97 during 1990-1991, and the follow-up heart rate readings were obtained during 1992-1993,

98 1994-1995, and 2001-2002. Heart rate parameters were the baseline and final heart rates.

99 Baseline heart rate was defined as the heart rate measured at the first examination, and the final

100 heart rate was defined as the last available heart rate during follow-up. The change in heart rate

101 was defined as the change in heart rates between examinations. When data were missing between

102 examinations, we omitted the missing heart rate. For example, if the second examination was

103 missed, then we calculated differences in the heart rates of the third and first examinations.

104 Group-based trajectory models (GBTMs) of heart rates were based on statistical analyses, and

105 individuals with similar patterns of changes in heart rate were grouped together. The heart rate

106 coefficient of variation (CV) was defined as the ratio of the standard deviation to the average

107 heart rate.

108

109 Outcomes

110 The primary outcome was all-cause mortality, and the secondary outcome was a composite of

111 coronary artery disease and cerebrovascular accidents (CVAs). Coronary artery disease was

112 defined as nonfatal myocardial infarction, fatal coronary artery disease, or hospitalization for

113 percutaneous coronary intervention or coronary artery bypass surgery, including hospitalization

114 for angina, and coronary angiography showing $>50 \%$ luminal stenosis. Fatal coronary artery

115 disease was considered to have occurred if hospital records confirmed fatal myocardial infarction

116 or if coronary heart disease was listed on the death certificate as the underlying and most

117 plausible cause of death. CVAs were defined as sudden neurological deficits of the vascular

118 origin that lasted for more than 24 hours. Primary and secondary outcomes identified during

119 follow-up visits were reviewed by physicians on the Committee of Mortality and Morbidity

120 within the study team. Four cardiologists reviewed the completed interview questionnaires,

121 medical records, and laboratory reports to determine whether each event met the protocol

122 established by the project steering committee. The study team invited all participants to have

123 biennial follow-up visits and examinations. Relatives of non-respondents were contacted to

124 obtain information on the health status of non-responsive individuals. Medical students were

125 responsible for administering the questionnaires and interviewing participants. We reviewed the

126 hospitalization and outpatient service records corresponding to the questionnaires. Moreover, we

127 regularly collected death certificate documents from the local health station and performed oral

128 interviews with relatives and family members about death events to confirm the causes of death.

129

130

131

132

133

Definitions of potential risk factors

134 alcohol habits, lifestyle choices, exercise frequency, socioeconomic status, and family history, 
135 were collected through questionnaires. Physical examinations were conducted by trained

136 physicians. Laboratory tests were conducted after a 12-hour fasting period. Blood pressure was

137 measured after $10 \mathrm{~min}$ of rest using a mercury sphygmomanometer. This measurement was

138 obtained with participants in a comfortable seated position, with their arms supported and

139 positioned at the level of the heart. Blood pressure was measured twice in the right arm. If the

140 readings varied by more than $10 \mathrm{mmHg}$, then an additional reading was performed. The average

141 of these blood pressure measurements was used as the final blood pressure.

142

143 Body mass index (BMI) was calculated as weight $(\mathrm{kg}) /$ height $^{2}\left(\mathrm{~m}^{2}\right)$. Diabetes mellitus (DM) was

144 defined as fasting glucose $\geq 126 \mathrm{mg} / \mathrm{dL}$ or as the use of oral hypoglycemic or insulin medication.

145 The estimated glomerular filtration rate (eGFR) was calculated using the Cockcroft-Gault

146 formula $[14,15]$. An eGFR $<60 \mathrm{~mL} / \mathrm{min} / 1.73 \mathrm{~m}^{2}$ indicated chronic kidney disease. Individuals

147 were defined as smokers or never smokers; the smokers group included current smokers and

148 those who had quit smoking. Regular exercise was defined as at least 20 min of physical activity

149 two or three times per week.

150

151

Statistical analysis

152 For descriptive analyses, continuous variables are described as mean ( \pm standard deviation), and categorical variables as numbers and percentages. Characteristics of demographic data across groups were compared using an analysis of variance for continuous variables and using chisquare test for categorical variables of sex, history of hypertension, DM, atrial fibrillation, smoking, and sports.

The GBTM analysis was performed using SAS software (version 9.4; SAS Institute, Cary, NC) with the Traj package to identify trajectory patterns of long-term changes in heart rate. We adopted the censored normal model because heart rate is a continuous variable. We initially assumed the trajectory patterns to be cubic and treated heart rate as a dependent variable and time as an independent variable. A repeated trajectory analysis was performed by changing the number of groups from two to five. The Bayesian information criterion was used to estimate the number of trajectory patterns. The number of groups with the least Bayesian information criterion was considered the appropriate one. After the number of groups was decided, we further tested each group of trajectories as linear, quadratic, or cubic to confirm the accurate graphical shape of change in heart rate by selecting the highest polynomial order to best characterize each trajectory group $[16,17]$. To test the robustness of our GBTM, we also analyzed trajectory patterns of only three repeated heart rate measurements obtained during 1990-1991, 1992-1993, and 1994-1995 and compared them with those of four repeated heart rate measurements.

Baseline and final heart rates were treated as both categorical and continuous variables in this study. To analyze the baseline and final heart rates as categorical variables, they were evaluated according to quartile. The change in heart rate was considered a continuous variable by calculating differences in heart rates between the first follow-up examination and baseline,

176 between the second and first follow-up examinations, and between the third and second follow-

179 A survival curve was calculated using the Kaplan-Meier method. For Cox regression analyses, 180 up examinations. The heart rate $\mathrm{CV}$ was considered a continuous variable. 
181 sex only. Model 2 was adjusted with all covariates. We used baseline covariates to adjust for the

182 baseline heart rate, GBTM of the heart rate, and CV of the heart rate, and we used time-

183 dependent covariates to adjust for the final heart rate and change in heart rate. These covariates

184 are established predictors of outcomes. Baseline covariates included age, sex, systolic blood

185 pressure (SBP), diastolic blood pressure (DBP), diabetes status, smoking, cholesterol, BMI, and

$186 \mathrm{eGFR}<60 \mathrm{~mL} / \mathrm{min} / 1.73 \mathrm{~m}^{2}$. The time-dependent covariates were age, BMI, cholesterol, diabetes

187 status, SBP, and DBP [11]. Statistical significance was defined as a two-tailed $\mathrm{P}<0.05$. All

188 analyses were performed with SAS version 9.4 (SAS Institute).

\section{Results}

190 Tables 1 and 2 show baseline characteristics of participants based on the quartile of the baseline

191 and final heart rates. No significant differences in baseline heart rate were found among the

192 groups. For the final heart rate, group 1 included participants with heart rate $<61$ beats per

193 minutes (bpm); group 2 comprised those with heart rate between 62 and 68 bpm; group 3

194 comprised those with heart rate between 69 and 75 bpm; and group 4 comprised those with heart

195 rate $>76 \mathrm{bpm}$. Participants in group 4 were likely to have the following characteristics: female

196 sex; higher BMI, cholesterol, and blood pressure; smoker; and diabetes. The four cubic

197 trajectories of resting heart rates derived from the GBTM are shown in Figure 2. We labeled

198 them according to the trajectory pattern: stable $(n=1,828 ; 61 \%)$, decreased $(n=155 ; 5 \%)$, mildly

199 increased $(n=958 ; 32 \%)$, and markedly increased $(n=74 ; 2 \%)$. Participants with stable, mildly

200 increased, and markedly increased heart rate patterns started with lower baseline heart rates,

201 whereas those with a decreased pattern started with higher baseline heart rates, but the trend

202 decreased later. The trend of an increased heart rate was the highest for the markedly increased

203

204

205

206

207

208

209

210

211

212

213

214

215

216

217

218

219

220

221

222

223

224

225 pattern and the lowest for the stable pattern. Table 3 shows baseline characteristics of each group. Sex, BMI, and cholesterol were significantly different among the groups. Participants in the markedly increased trajectory pattern group were likely to have higher BMI, cholesterol, and DBP than those in the other groups. Participants in the decreased trajectory pattern group were likely to be women, have a history of hypertension, and have higher SBP, and those with stable trajectory pattern were likely to be men, be smokers, have lower BMI and cholesterol levels, and have lower SBP and DBP.

Kaplan-Meier survival curves for all-cause mortality as well as the composite of outcomes of coronary artery disease and CVA according to the four heart rate trajectory patterns are shown in Figures 3 and 4. The markedly increased trajectory pattern showed the worst survival rate, and the log-rank test results for all-cause mortality among the four patterns of heart rate change were significant $(p=0.011)$. For the composite outcome of coronary artery disease and CVA, no significant differences were found among the four heart rate trajectory patterns $(p=0.77)$. The adjusted hazard ratios of primary and secondary outcomes for participants with markedly increased trajectory pattern were 1.80 (95\% confidence interval [CI], 1.18-2.76; $p=0.007)$ and $1.45(95 \%$ CI, $0.67-3.12 ; p=0.34)$, respectively, compared with those with the stable trajectory pattern (Table 4). For GBTM of the heart rate with three repeated heart rate measurements, four heart rate trajectories were identified, and their trends were similar to the results of the four repeated heart rate measurements. The adjusted hazard ratios of the primary and secondary outcomes for participants with markedly increased trajectory pattern were 1.62 (95\% CI, 1.10 $2.38 ; p=0.015)$ and $0.87(95 \% \mathrm{CI}, 0.40-1.88 ; p=0.71)$ (Table 5), respectively. 


\section{Discussion}

227 Main findings

228 The study demonstrated an association between heart rate trajectories and all-cause mortality, but

229 no association was evident between heart rate trajectories and cardiovascular events. Our study

230 was primarily based on the analysis of heart rate in sinus rhythm. Participants with atrial

231 fibrillation were excluded because previous literature showed that the impact of heart rate on

232 adverse outcomes differed among patients with sinus rhythm and those with atrial fibrillation

$233[18,19]$. We also excluded participants using antihypertensive medications due to their strong

234 influence on heart rate [20,21]. Determining the risk of all-cause mortality from the baseline

235 heart rate is sometimes arbitrary, particularly when the change in heart rate or variation with time

236 is high in the cohort population. The baseline heart rate was not a good surrogate marker in this

237 study. Based on the heart rate trajectories, only half of the participants had a stable heart rate,

238 whereas the other half had a heart rate variation over $15 \%$ in one decade.

239

240 Heart rate trajectories with time-dependent covariates adjustment will predict outcomes better in

241

242

243

244

245

246

247

248

249

250

251

252

253

254

255

256

257

258

259

260

261

262

263

264

265

266

267

268

269

270

271 this situation. Participants with a decreased trajectory pattern started with the highest baseline heart rate among the groups. The risk of all-cause mortality in patients with a decreased trajectory pattern was not higher than that in patients with a stable trajectory pattern. Although participants with a decreased trajectory pattern started with higher baseline heart rates, the heart rate trajectory of the decreased pattern continued to decrease over time. The detrimental effect of a high baseline heart rate was diminished by the decrease in heart rate over time. However, participants with a stable trajectory pattern and those with markedly increased trajectory pattern started with similar baseline heart rates, but the heart rate trajectory of the markedly increased pattern increased over time. An approximate 50\% increase in heart rate was found from the baseline in 2 years. The risk of all-cause mortality for those with markedly increased trajectory pattern was significantly higher than for those with stable trajectory pattern. The faster the heart rate increases, the higher the all-cause mortality rate. This finding confirmed that the heart rate trajectory could be a more sensitive predictor of adverse outcomes than a single heart rate measurement.

In our study, there was a gap between the last heart rate measurement and final outcomes. This temporal relationship demonstrates that the observed associations were not due to reverse causation. Further, we compared the results of GBTM for heart rate with three and four repeated heart rate measurements and found that the trajectories were very close and that the markedly increased trajectory pattern was associated with a significantly higher risk of all-cause mortality than the stable pattern in both models. This finding confirmed the reliability of our heart rate group classification and showed the possibility to predict long-term heart rate trajectory patterns with earlier repeated heart rate measurements.

Compared with the GBTM for heart rate, the change in heart rate did not correlate well with adverse outcomes. This finding may have been caused by the change in heart rate being considered as a continuous variable because it was difficult to predict a complex and non-linear trend for the risk of heart rate outcomes. The heart rate $\mathrm{CV}$ showed the extent of variability in relation to the mean heart rate but lacked information regarding how the heart rate changed with time; therefore, it correlated poorly with outcomes. 
272 For the secondary outcomes, there are several possible reasons for the insignificant results. First,

273 a low events rate for secondary outcomes was responsible for the low statistical power in our

274 population. Second, considering the effect size of trajectories on secondary outcomes, our

275 sample size may have been too small for the analysis.

276

277

\section{Comparisons with previous studies}

278 Previous literature emphasized the role of resting heart rate as a predictor of adverse outcomes,

279

280

281

282

283

284

285

286

287

288

289

290

291

292

293

294

295

296

297

298

299

300

301

302

303

304

305

306

307

308

309

310

311

312

313

314

315

316 Heart rate, as a component of vital signs, is underrated in the long term. The most widely 317 accepted "normal heart rate" ranges from 60 to $100 \mathrm{bpm}$ [31]. In the present study, most and resting heart rate was regarded as an indicator of general health [22]. In one large cohort investigating the association of resting heart rate with cause-specific mortality, the authors found that heart rate over a decade was associated with a risk of death from cancer, cardiovascular diseases, and other causes. The hazard ratio of mortality was higher for cardiovascular diseases than for cancer. Our study used coronary artery diseases and stroke as secondary outcomes, which included mostly death from cardiovascular diseases. The impact of heart rate trajectory with outcomes was consistent with findings from a previous research; however, due to limited numbers of events, the results were not statistically significant [23].

Some studies reported that heart rate achieved during follow-up examinations was a better predictor of risk than the baseline heart rate $[4,18]$. Other studies showed that the change in heart rate was positively correlated with all-cause mortality and non-fatal cardiovascular events [2326]. Clinical benefits were also reported to be associated with heart rate reductions [21,27]. In the present study, the heart rate trajectory consisted of not only the baseline heart rate or a single measurement of heart rate but also the change in heart rate over time. By recognizing the patterns of change in heart rate rather than the absolute numerical heart rate value, adverse outcomes can be predicted intuitively. Chen et al. found that heart rate trajectories could predict artery stiffness. Compared with that study, we followed our participants for years after establishing the heart rate trajectory model to demonstrate the relationship between trajectories and all-cause mortality [12].

Participants in the markedly increased heart rate pattern group were likely to have higher BMI, DBP, and cholesterol. Several studies showed the association of these risk factors with heart rate. Lower BMI is associated with cardiorespiratory fitness. Regular exercise can improve cardiorespiratory fitness, which was proven by some studies to decrease resting heart rate [28]. Resting heart rate is an indicator of autonomic function. Autonomic dysfunction leads to a higher resting heart rate in hypertension, hyperlipidemia, and metabolic syndrome [29,30]. However, further studies are necessary to clarify the role of heart rate with these risk factors and adverse outcomes.

\section{Implications for clinical management}

Our study was based on a community cohort, and most participants were healthy individuals. Although the external validity of our study was not tested, our results highlighted the possibility of investigating the heart rate not only using a single measurement but also using trajectory patterns. In addition, our study demonstrated the utility of repeated heart rate measurements for risk assessment. 
318 participants' heart rates were in the normal heart rate range; however, the markedly increased

319 trajectory pattern was associated with a higher risk of all-cause mortality than the stable

320 trajectory pattern. The importance of heart rate trajectory patterns cannot be overstressed.

321 Because wearable devices have become increasingly popular, they may be helpful for recording

322 large amounts of heart rate data, thereby allowing data analysis algorithms to define the

323 trajectory more accurately and easily.

324

325

326

327

328

329

330

331

332

333

334

335

336

337

338

339

340

\section{Study strengths and limitations}

This study had three major strengths. First, our study was based on a large sample size and included a long-term follow-up cohort study, both of which could have reduced the possibility of selection bias. Second, this study used several heart rate parameters, including the GBTM of heart rate, to estimate the effects of changes in heart rate on adverse outcomes. Third, our heart rate measurements were based on the ECG not the pulse rate. Participants would rest for 10 minutes before the heart rate was measured. This type of standardization enhanced the accuracy of heart rate measurements. This study also had limitations. Our results were based on the GBTM, and group identifications were not certain. The GBTM may identify additional groups to accommodate non-normality in the data rather than true latent groups. Other limitations include a large attrition between the follow-up visits, lack of medication information collected at the follow-up visits, oversimplified grading of physical activity, lack of updated information about smoking status and kidney function, and lack of specific cause of death. In addition, some residual confounders, such as inflammation, infection, weight gain, pregnancy, thyroid function, and other metabolic factors, were not well adjusted in the study.

\section{Conclusions}

341

342

343

344

345

346

\section{7}

348

349

350

351

352

353

354

355

356

357

This study demonstrated the importance of identifying specific heart rate trajectory patterns and elucidating their associations with all-cause mortality, coronary artery disease, and CVA in a community-based population. The markedly increased trajectory pattern was associated with a much higher risk of all-cause mortality than the stable trajectory pattern, even when the heart rate was in the normal range.

\section{Acknowledgments}

We would like to thank the cardiologists, nurses, and medical students at National Taiwan University Hospital for their assistance with this study.

\section{References}

1. Kannel, W.B., Kannel, C., Paffenbarger, R.S., Cupples, L.A. Heart rate and cardiovascular mortality: the Framingham Study. Am Heart J 1987, 113, 1489-1494.

2. Aune, D., Sen, A., O’Hartaigh, B., Janszky, I., Romundstad, P.R., Tonstad, S., Vatten, L. Resting heart rate and the risk of cardiovascular disease, total cancer, and all-cause mortality - a systematic review and dose-response meta-analysis of prospective studies. Nutr Metab

Peer) reviewing PDF | (2019:10:42578:2:1:NEW 20 Mar 2020) 
360

361

362

363

364

365

366

367

368

369

370

371

372

373

374

375

376

377

378

379

380

381

382

383

384

385

386

387

388

389

390

391

392

393

394

395

396

397

398

399

400

401

402

403

Cardiovasc Dis 2017, 27, 504-517.

3. Zhang, D., Shen, X., Qi, X. Resting heart rate and all-cause and cardiovascular mortality in the general population: a meta-analysis. CMAJ 2016, 188, E53-E63.

4. Paul, L., Hastie, C.E., Li, W.S., Harrow, C., Muir, S., Connell, J.M., Dominiczak, A.F., McInnes, G.T., Padmanabhan, S. Resting heart rate pattern during follow-up and mortality in hypertensive patients. Hypertension 2010, 55, 567-574.

5. Diaz, A., Bourassa, M., Guertin, M., Tardif, J. Long-term prognostic value of resting heart rate in patients with suspected or proven coronary artery disease. Eur Heart J 2005, 26, 967-974.

6. Chioncel, O., Mebazaa, A., Harjola, V., Coats, A., Piepoli, M., Crespo-Leiro, M., Laroche, C., Seferovic, P., Anker, S., Ferrari, R., Ruschitzka, F., Lopez-Fernandez, S., Miani, D.,

Filippatos, G., Maggioni, A. Clinical phenotypes and outcome of patients hospitalized for acute heart failure: the ESC Heart Failure Long-Term Registry. Eur J Heart Fail 2017, 19, 1242-1254. 7. Jouven, X., Empana, J., Escolano, S., Buyck, J., Tafflet, M., Desnos, M., Ducimetiere, P. Relation of heart rate at rest and long-term ( $>20$ years) death rate in initially healthy middle-aged men. Am J Cardiol 2009, 103, 279-283.

8. Cooney, M.T., Vartiainen, E., Laatikainen, T., Juolevi, A., Dudina, A., Graham, I.M. Elevated resting heart rate is an independent risk factor for cardiovascular disease in healthy men and women. Am Heart $J$ 2010, 159, 612-619 e3.

9. Tverdal, A., Hjellvik, V., Selmer, R. Heart rate and mortality from cardiovascular causes: a 12 year follow-up study of 379,843 men and women aged 40-45 years. Eur Heart $J$ 2008, 29, $2772-2781$.

10. Nauman, J., Janszky, I., Lars, J.V., Ulrik, W. Temporal changes in resting heart rate and deaths from ischemic heart disease. JAMA 2011, 306, 2579-2587.

11. Vazir, A., Claggett, B., Cheng, S., Skali, H., Shah, A., Agulair, D., Ballantyne, C.M., Vardeny, O., Solomon, S.D. Association of resting heart rate and temporal changes in heart rate with outcomes in participants of the atherosclerosis risk in communities study. JAMA Cardiol 2018, 3, 200-206.

12. Chen, S., Li, W., Jin, C., Vaydia, A., Gao, J., Yang, H., Wu, S., Gao, X. Resting heart rate trajectory pattern predicts arterial stiffness in a community-based Chinese cohort. Arterioscler Thromb Vasc Biol 2017, 37, 359-364.

13. Lee, Y.T., Lin, R.S., Sung, F.C., Yang, C.Y., Chien, K.L., Chen, W.J., Su, T.C., Hsu, H.C., Huang, Y.C. Chin-Shan community cardiovascular cohort in Taiwan-baseline data and five-year follow-up morbidity and mortality. J Clin Epidemiol 2000, 53, 838-846.

14. Davies, M.J., D'Alessio, D.A., Fradkin, J., Kernan, W.N., Mathieu, C., Mingrone, G., Rossing, P., Tsapas, A., Wexler, D.J., Buse, J.B. Management of hyperglycemia in type 2 diabetes, 2018. A consensus report by the American Diabetes Association (ADA) and the European Association for the Study of Diabetes (EASD). Diabetes Care 2018, 41, 2669-2701. 15. O'Hare, A.M., Choi, A.I., Bertenthal, D., Bacchetti, P., Garg, A.X., Kaufman, J.S., Walter, L.C., Mehta, K.M., Steinman, M.A., Allon, M., McClellan, W.M., Landefeld, C.S. Age affects outcomes in chronic kidney disease. J Am Soc Nephrol 2007, 18, 2758-2765.

16. Nagin, D.S. Analyzing developmental trajectories: a semiparametric, group-based approach. Psychol Methods 1999, 4, 139-157.

17. Nagin, D.S., Odgers, C.L. Group-based trajectory modeling in clinical research. Annu Rev Clin Psychol 2010, 6, 109-138.

18. Kotecha, D., Flather, M.D., Altman, D.G., Holmes, J., Rosano, G., Wikstrand, J., Packer, M., Coats, A.J.S., Manzano, L., Bohm, M., van Veldhuisen, D.J., Andersson, B., Wedel, H., von 
404 Lueder, T.G., Rigby, A.S., Hjalmarson, A., Kjekshus, J., Cleland, J.G.F. Beta-Blockers in Heart 405 Failure Collaborative Group. Heart rate and rhythm and the benefit of beta-blockers in patients 406 with heart failure. J Am Coll Cardiol 2017, 69, 2885-2896.

407 19. Laskey, W.K., Alomari, I., Cox, M. Schulte, P.J., Zhao, X., Hernandez, A.F., Heidenreich, 408 P.A., Eapen, Z.J., Yancy, C., Bhatt, D.L., Fonarow, G.C. Heart rate at hospital discharge in 409 patients with heart failure is associated with mortality and rehospitalization. J Am Heart Assoc 410 2015, 4, pii: e001626. doi: 10.1161/JAHA.114.001626.1-19.

411 20. Whelton, P.K., Carey, R.M., Aronow, W.S., Casey, D.E., Jr., Collins, K.J., Dennison 412 Himmelfarb, C., DePalma, S.M., Gidding, S., Jamerson, K.A., Jones, D.W., MacLaughlin, E.J., 413 Muntner, P., Ovbiagele, B., Smith, S.C., Jr., Spencer, C.C., Stafford, R.S., Taler, S.J., Thomas, 414 R.J., Williams, K.A., Sr, Williamson, J.D., Wright, J.T., Jr. 2017

415 ACC/AHA/AAPA/ABC/ACPM/AGS/APhA/ASH/ASPC/NMA/PCNA Guideline for the 416 prevention, detection, evaluation, and management of high blood pressure in adults: a report of 417 the American College of Cardiology/American Heart Association Task Force on clinical practice 418 guidelines. Hypertension 2018, 71, e13-e115.

419 21. McAlister, F.A., Wiebe, N., Ezekowitz, J.A., Leung, A.A., Armstrong, P.W. Meta-analysis: 420 beta-blocker dose, heart rate reduction, and death in patients with heart failure. Ann Intern Med 421 2009, 150, 784-794.

422 22. Lindgren, M., Robertson, J., Adiels, M., Schaufelberger, M., Åberg, M., Torén, K., Waern, 423 M., Åberg, N.D., Rosengren, A. Resting heart rate in late adolescence and long term risk of cardiovascular disease in Swedish men. Int J Cardiol 2018, 259, 109-115.

23. Seviiri M., Lynch B., Hodge A., Yang Y., Liew D., English D., Giles G., Milne R., Dugue P.A. Resting heart rate, temporal changes in resting heart rate, and overall and cause specific mortality. Heart 2018, 104, 1076-1085

429

430

431 24. Takahama, H., Yokoyama, H., Kada, A., Sekiguchi, K., Fujino, M., Funada, A., Amaki, M., Hasegawa, T., Asakura, M., Kanzaki, H., Anzai, T., Kitakaze, M. Extent of heart rate reduction during hospitalization using beta-blockers, not the achieved heart rate itself at discharge, predicts the clinical outcome in patients with acute heart failure syndromes. J Cardiol 2013, 61, 58-64.

433

434

435 25. Vazir, A., Claggett, B., Pitt, B., Anand, I., Sweitzer, N., Fang, J., Fleg, J., Rouleau, J., Shah, S., Pfeffer, M.A., Solomon, S.D. Prognostic importance of temporal changes in resting heart rate in heart failure and preserved ejection fraction: from the TOPCAT Study. JACC Heart Fail 2017, 5, 782-791.

26 Kitai, T., Grodin, J., Mentz, R., Hernandez, A., Butler, J., Metra, M., McMurray, J., Armstrong, P., Starling, R., O’Connor, C., Swedberg, K., Tang, W. Insufficient reduction in heart rate during hospitalization despite beta-blocker treatment in acute decompensated heart failure: insights from the ASCEND-HF trial. Eur J Heart Fail 2017, 19, 241-249.

441

442 27. Swedberg, K., Komajda, M., Böhm, M., Borer, J.S., Ford, I., Dubost-Brama, A., Lerebours, G., Tavazzi, L. Ivabradine and outcomes in chronic heart failure (SHIFT): a randomised placebocontrolled study. Lancet 2010, 376, 875-885.

443 28. Quan, H.L., Blizzard, C.L., Sharman, J.E., Magnussen, C.G., Dwyer, T., Raitakari, O., 444 Cheung, M., Venn, A.J. Resting heart rate and the association of physical fitness with carotid 445 artery stiffness. Am J Hypertens 2014, 27, 65-71.

446 29. Inoue, T., Iseki, K., Iseki, C., Kinjo, K., Ohya, Y., Takishita, S. Higher heart rate predicts the 447 risk of developing hypertension in a normotensive screened cohort. Circ J 2007, 71, 1755-1760. 448 30. Sun, K., Liu, Y., Dai, M., Li, M., Yang, Z., Xu, M., Xu, Y., Lu, J., Chen, Y., Liu, J., Ning, 449 G., Bi, Y. Accessing autonomic function: can early screen metabolic syndrome. PLoS ONE 
$450 \quad$ 2012, 7, e43449.

451 31. Fleming, S., Thompson, M., Stevens, R., Heneghan, C., Plüddemann, A., Maconochie, I., 452 Tarassenko, L., Mant, D. Normal ranges of heart rate and respiratory rate in children from birth 453 to 18 years: a systematic review of observational studies. Lancet 2011, 377, 1011-1018. 
Figure 1

Flow diagram of the cohort selection process.

The diagram shows the events, exclusion criteria, and the number of participants in each visit. 


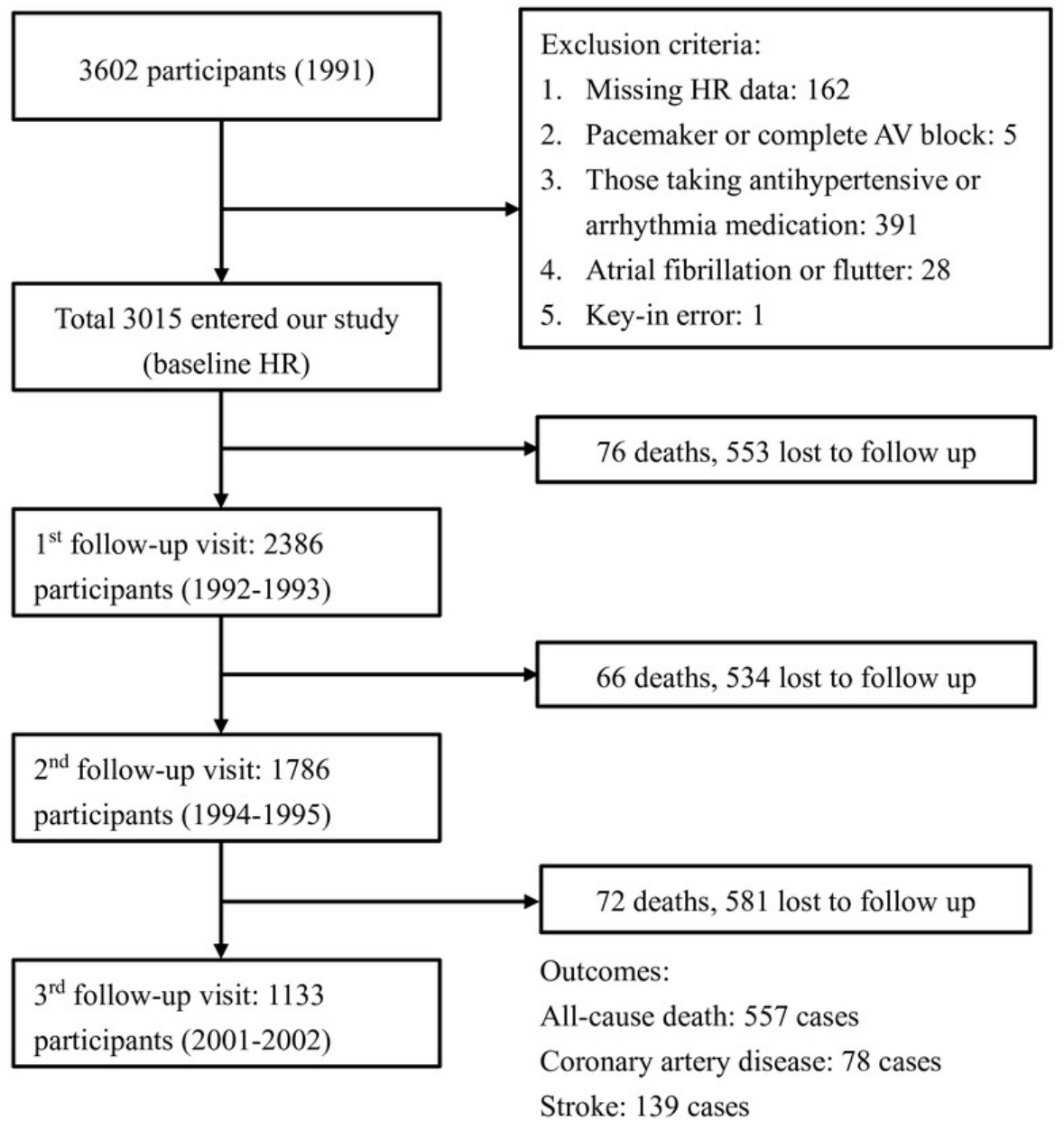


Figure 2

Four heart rate trajectory patterns identified by the group-based trajectory model.

The figure shows four trajectory patterns in four different colors. The red line indicates markedly increased pattern. The orange line indicates mildly increased pattern. The green line indicates decreased pattern. The blue line indicates stable pattern. The dotted line is the $95 \%$ confidence interval of heart rate.

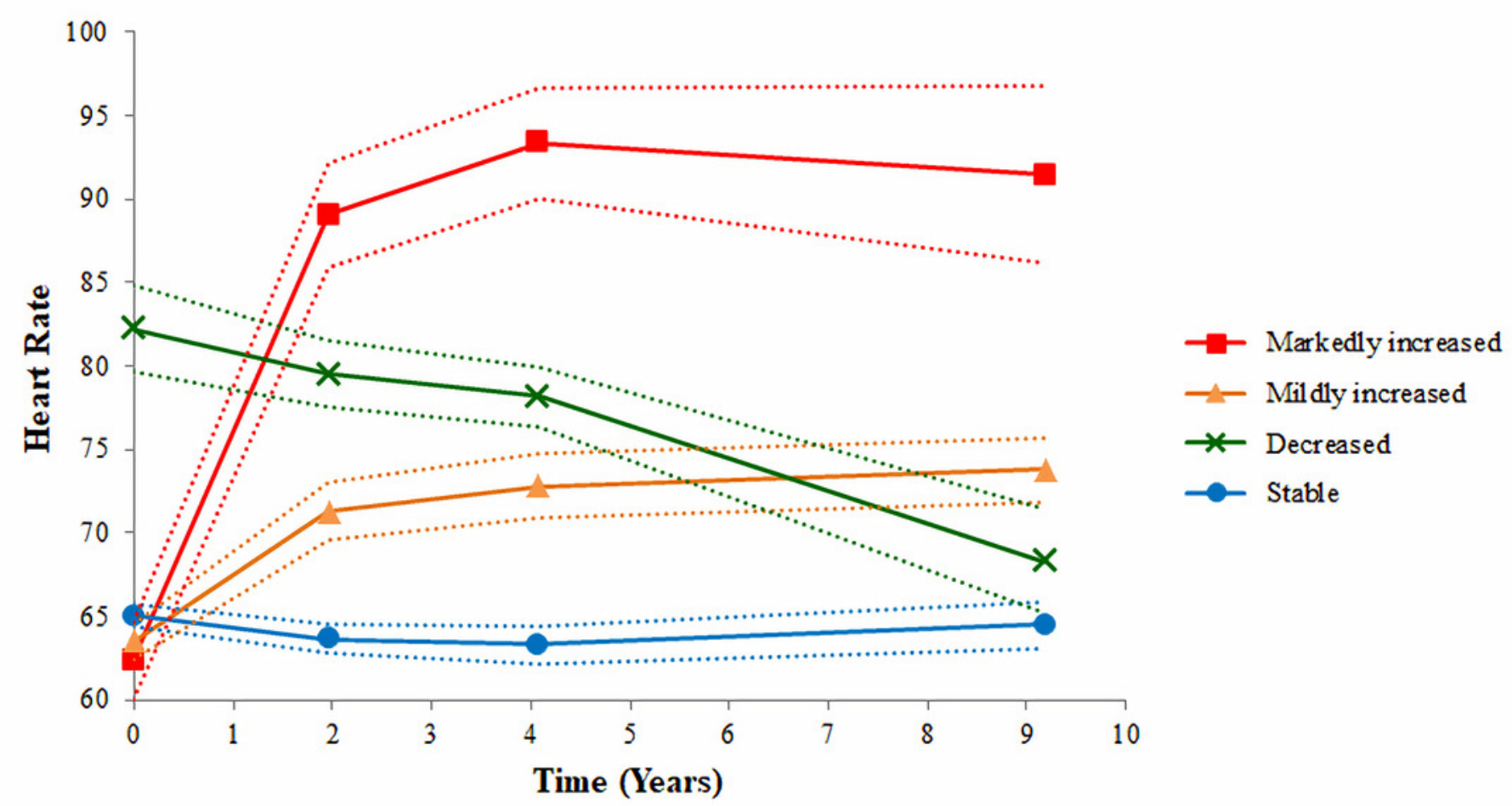


Figure 3

Kaplan-Meier survival curves for all-cause mortality based on group-based trajectory model of the heart rate.

The red line indicates the survival curve of markedly increased pattern. The orange line indicates the survival curve of mildly increased pattern. The green line indicates the survival curve of decreased pattern. The blue line indicates the survival curve of stable pattern.

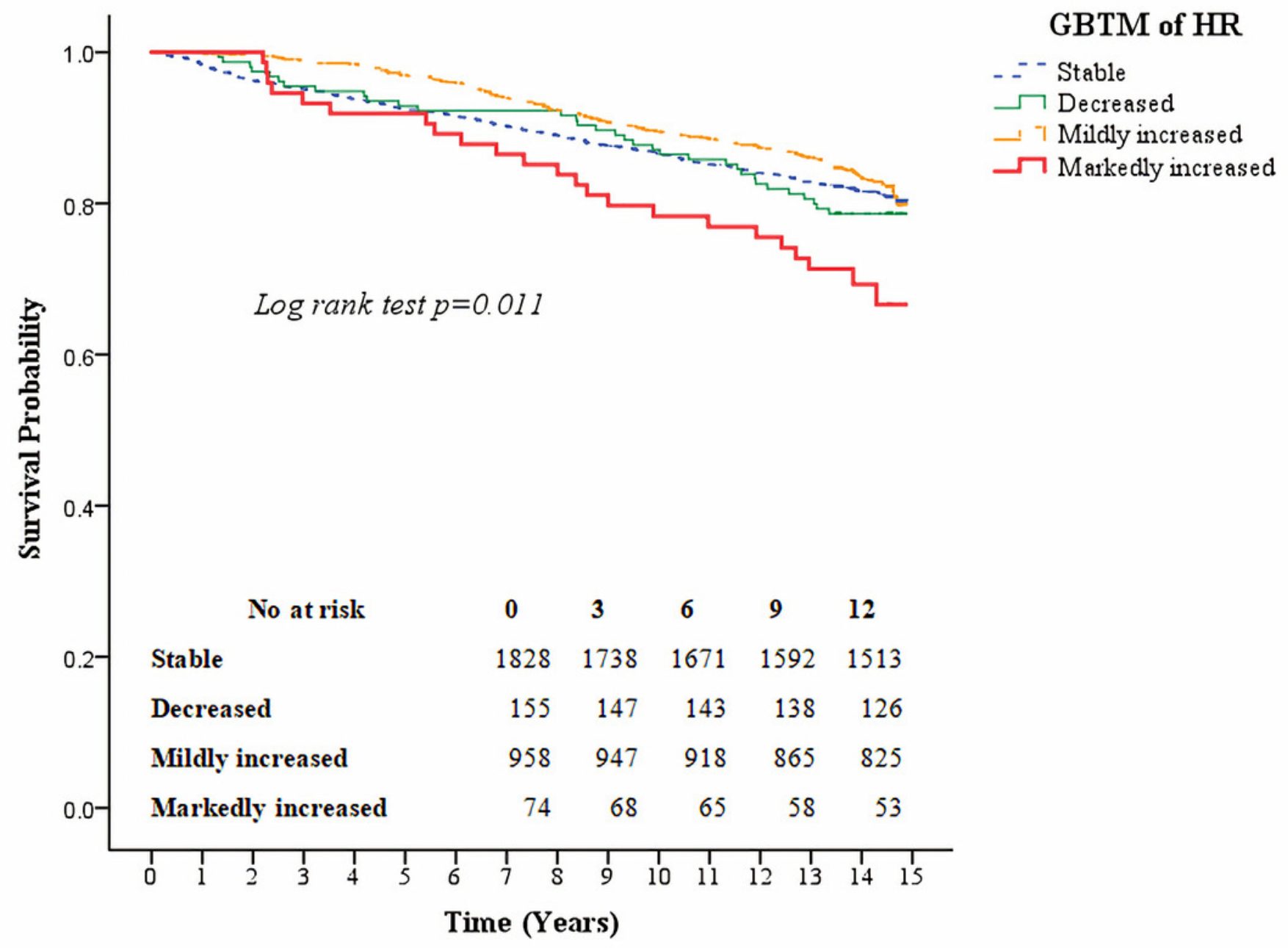




\section{Figure 4}

Kaplan-Meier survival curves for the composite outcome of coronary artery disease and cerebral vascular accidents based on the group-based trajectory model of the heart rate.

The red line indicates the survival curve of markedly increased pattern. The orange line indicates the survival curve of mildly increased pattern. The green line indicates the survival curve of decreased pattern. The blue line indicates the survival curve of stable pattern.

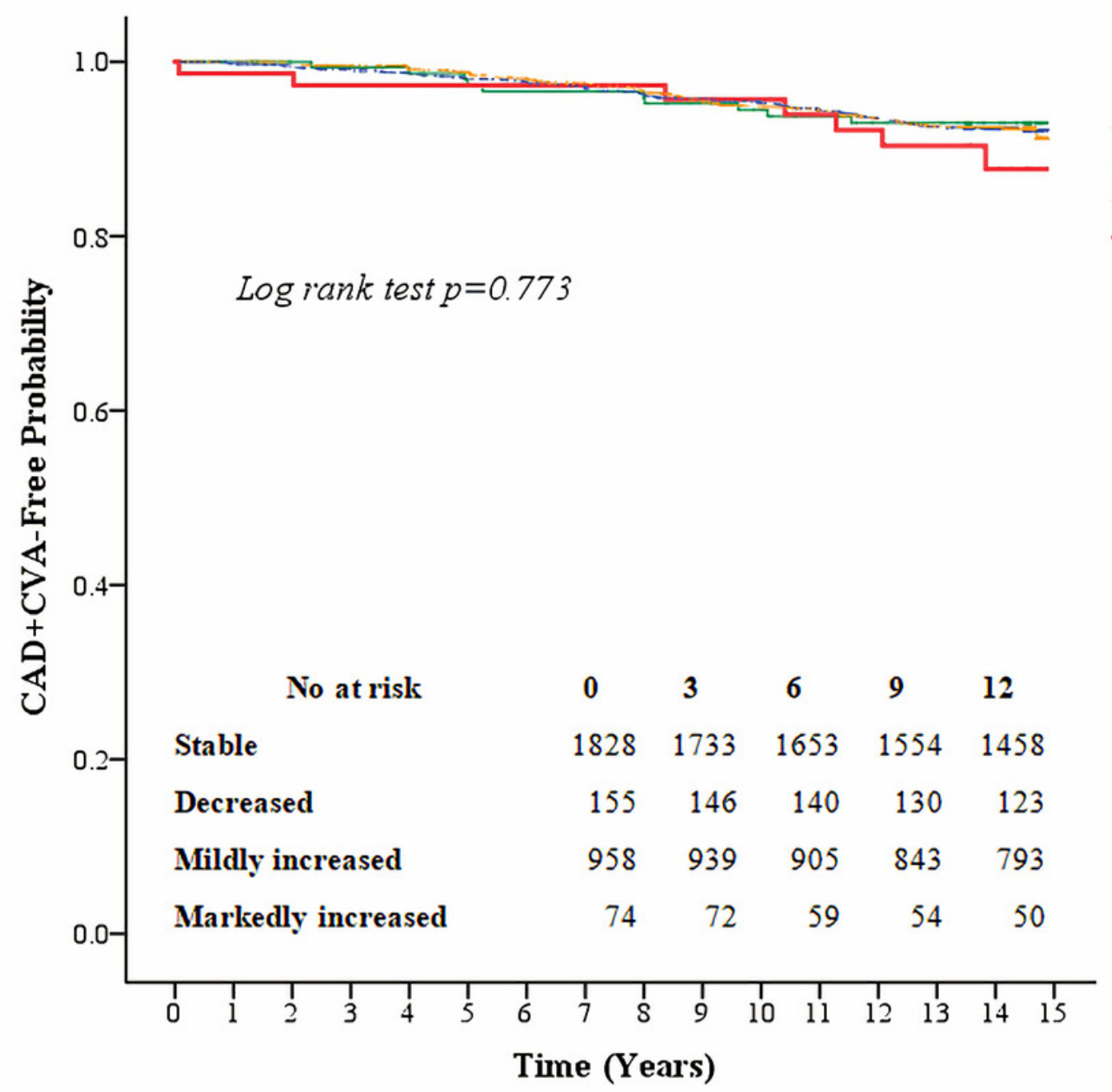




\section{Table 1 (on next page)}

Baseline characteristics of participants based by baseline heart rate quartile 


\begin{tabular}{|c|c|c|c|c|c|}
\hline $\begin{array}{l}\text { Number } \\
\text { Mean (SD) }\end{array}$ & $\begin{array}{c}\text { Group 1 } \\
\text { (Baseline HR } \\
\leq 58) \\
(\mathrm{n}=766)\end{array}$ & $\begin{array}{c}\text { Group } 2 \\
\text { (59 } \leq \text { Baseline } \\
\text { HR } \leq 64) \\
(n=762)\end{array}$ & $\begin{array}{c}\text { Group } 3 \\
\text { (65 } \leq \text { Baseline } \\
H R \leq 72) \\
(n=809)\end{array}$ & $\begin{array}{c}\text { Group } 4 \\
\text { (Baseline HR } \\
\geq 73) \\
(\mathrm{n}=678)\end{array}$ & P-value \\
\hline Age (years) & $54.0 \pm 11.9$ & $53.7 \pm 12.3$ & $53.6 \pm 12.3$ & $54.3 \pm 12.4$ & 0.68 \\
\hline Sex (female) & $407(53.1 \%)$ & $396(52.0 \%)$ & $416(51.4 \%)$ & $355(52.4 \%)$ & 0.92 \\
\hline BMI $\left(\mathrm{kg} / \mathrm{m}^{2}\right)$ & $23.3 \pm 3.5$ & $23.3 \pm 3.2$ & $23.2 \pm 3.3$ & $23.2 \pm 3.3$ & 0.86 \\
\hline Cholesterol (mg/dL) & $195.9 \pm 46.3$ & $197.7 \pm 43.8$ & $196.5 \pm 42.7$ & $195.9 \pm 44.9$ & 0.85 \\
\hline DM & $98(12.9 \%)$ & $73(9.6 \%)$ & $102(12.6 \%)$ & $85(12.6 \%)$ & 0.16 \\
\hline Hypertension & $150(19.7 \%)$ & $168(22.2 \%)$ & 155 (19.4\%) & $142(21.0 \%)$ & 0.51 \\
\hline Smoking & $261(34.1 \%)$ & $287(37.7 \%)$ & $311(38.4 \%)$ & $246(36.3 \%)$ & 0.30 \\
\hline Alcohol & $217(28.3 \%)$ & $233(30.6 \%)$ & $250(30.9 \%)$ & $219(32.3 \%)$ & 0.42 \\
\hline Exercise & $119(15.5 \%)$ & $109(14.3 \%)$ & $104(12.9 \%)$ & $96(14.2 \%)$ & 0.51 \\
\hline SBP (mmHg) & $121.6 \pm 17.7$ & $122.7 \pm 18.4$ & $122.0 \pm 18.7$ & $123.1 \pm 17.6$ & 0.36 \\
\hline DBP (mmHg) & $75.7 \pm 10.5$ & $76.5 \pm 10.6$ & $75.4 \pm 10.3$ & $75.9 \pm 9.8$ & 0.22 \\
\hline eGFR $<60 \mathrm{~mL} / \mathrm{min}$ & $172(22.7 \%)$ & $162(21.4 \%)$ & $176(22.0 \%)$ & $165(24.4 \%)$ & 0.55 \\
\hline All-cause mortality & $141(18.4 \%)$ & $136(17.9 \%)$ & $151(18.7 \%)$ & $129(19 \%)$ & 0.95 \\
\hline $\begin{array}{l}\text { Coronary artery disease and } \\
\text { cerebral vascular accident }\end{array}$ & $55(7.2 \%)$ & $64(8.4 \%)$ & $54(6.7 \%)$ & $40(5.9 \%)$ & 0.30 \\
\hline
\end{tabular}

Abbreviations: BMI, body mass index; DBP, diastolic blood pressure; DM, diabetes mellitus; eGFR, estimated glomerular filtration rate; $\mathrm{HR}$, heart rate; SBP, systolic blood pressure; SD, standard deviation 


\section{Table 2 (on next page)}

Baseline characteristics of participants by final heart rate quartile. 


\begin{tabular}{|c|c|c|c|c|c|}
\hline $\begin{array}{l}\text { Number } \\
\text { Mean (SD) }\end{array}$ & $\begin{array}{c}\text { Group } 1 \\
\text { (Final HR } \leq 61) \\
(n=788)\end{array}$ & $\begin{array}{c}\text { Group } 2 \\
(62 \leq \text { Final } \\
H R \leq 68) \\
(n=791)\end{array}$ & $\begin{array}{c}\text { Group } 3 \\
(69 \leq \text { Final } \\
H R \leq 75) \\
(n=701)\end{array}$ & $\begin{array}{c}\text { Group } 4 \\
\text { (Final HR } \geq 76) \\
(n=735)\end{array}$ & P-value \\
\hline Age (years) & $59.5 \pm 12.2$ & $58.5 \pm 12.3$ & $58.4 \pm 12.0$ & $59.7 \pm 12.2$ & 0.09 \\
\hline Sex (female) & $346(43.9 \%)$ & $415(52.5 \%)$ & $399(56.9 \%)$ & $414(56.3 \%)$ & $<0.001$ \\
\hline BMI $\left(\mathrm{kg} / \mathrm{m}^{2}\right)$ & $23.3 \pm 3.2$ & $23.6 \pm 3.3$ & $23.7 \pm 3.7$ & $24.0 \pm 3.9$ & 0.001 \\
\hline Cholesterol (mg/dL) & $199.5 \pm 43.5$ & $198.1 \pm 42.4$ & $202.9 \pm 43.9$ & $203.5 \pm 42.7$ & 0.06 \\
\hline DM & $95(13.3 \%)$ & $93(12.9 \%)$ & $116(18.3 \%)$ & $164(25.2 \%)$ & $<0.001$ \\
\hline Hypertension & $126(19.0 \%)$ & $121(18.3 \%)$ & $142(24.6 \%)$ & $164(28.7 \%)$ & $<0.001$ \\
\hline Smoking & $337(42.8 \%)$ & $275(34.8 \%)$ & $231(33.0 \%)$ & $262(35.7 \%)$ & $<0.001$ \\
\hline Alcohol & $264(33.5 \%)$ & $241(30.5 \%)$ & $201(28.7 \%)$ & $213(29 \%)$ & 0.16 \\
\hline Exercise & $119(15.1 \%)$ & $117(14.8 \%)$ & $95(13.6 \%)$ & $97(13.2 \%)$ & 0.66 \\
\hline SBP (mmHg) & $123.6 \pm 20.6$ & $124.6 \pm 19.5$ & $126.5 \pm 19.5$ & $128.9 \pm 19.7$ & $<0.001$ \\
\hline DBP (mmHg) & $74.5 \pm 10.9$ & $75.2 \pm 11.4$ & $77.2 \pm 11.8$ & $78.9 \pm 11.7$ & $<0.001$ \\
\hline eGFR $<60 \mathrm{~mL} / \mathrm{min}$ & $193(24.7 \%)$ & $161(20.6 \%)$ & $154(22.0 \%)$ & $167(22.9 \%)$ & 0.26 \\
\hline All-cause mortality & $144(18.3 \%)$ & $139(17.6 \%)$ & $118(16.8 \%)$ & $156(21.2 \%)$ & 0.15 \\
\hline $\begin{array}{l}\text { Coronary artery disease and } \\
\text { cerebral vascular accident }\end{array}$ & $63(8 \%)$ & $49(6.2 \%)$ & $50(7.1 \%)$ & $51(6.9 \%)$ & 0.58 \\
\hline
\end{tabular}

Abbreviations: BMI, body mass index; DBP, diastolic blood pressure; DM, diabetes mellitus; eGFR, estimated glomerular filtration rate; HR, heart rate; SBP, systolic blood pressure; SD, standard deviation 


\section{Table 3(on next page)}

Baseline characteristics of participants based on the GBTM of the heart rate. 


\begin{tabular}{|c|c|c|c|c|c|}
\hline $\begin{array}{l}\text { Number } \\
\text { Mean (SD) }\end{array}$ & $\begin{array}{c}\text { Stable } \\
(\mathrm{n}=1,828)\end{array}$ & $\begin{array}{c}\text { Decreased } \\
\qquad(\mathrm{n}=155)\end{array}$ & $\begin{array}{c}\text { Mildly } \\
\text { increased } \\
(\mathrm{n}=958)\end{array}$ & $\begin{array}{c}\text { Markedly } \\
\text { increased } \\
(\mathrm{n}=74)\end{array}$ & P-value \\
\hline Age (years) & $54 \pm 12.1$ & $55 \pm 13.4$ & $53.3 \pm 12.2$ & $55.3 \pm 12.3$ & 0.23 \\
\hline Sex (female) & $880(48.1 \%)$ & $92(59.4 \%)$ & $559(58.4 \%)$ & $43(58.1 \%)$ & $<0.001$ \\
\hline BMI $\left(\mathrm{kg} / \mathrm{m}^{2}\right)$ & $23 \pm 3.1$ & $23.1 \pm 3.4$ & $23.7 \pm 3.6$ & $24.1 \pm 4.4$ & $<0.001$ \\
\hline Cholesterol (mg/dL) & $194.1 \pm 44.1$ & $199.4 \pm 44.9$ & $199.8 \pm 44.5$ & $207.6 \pm 45.5$ & 0.001 \\
\hline DM & $197(10.8 \%)$ & $18(11.8 \%)$ & $130(13.6 \%)$ & $13(17.6 \%)$ & 0.08 \\
\hline Hypertension & $348(19.2 \%)$ & $44(28.6 \%)$ & $205(21.5 \%)$ & $18(24.3 \%)$ & 0.025 \\
\hline Smoking & $713(39 \%)$ & $53(34.2 \%)$ & $314(32.8 \%)$ & $25(33.8 \%)$ & 0.011 \\
\hline Alcohol & $590(32.3 \%)$ & $47(30.3 \%)$ & $259(27 \%)$ & $23(31.1 \%)$ & 0.041 \\
\hline Sport & $265(14.5 \%)$ & $19(12.3 \%)$ & $134(14 \%)$ & $10(13.5 \%)$ & 0.88 \\
\hline SBP (mmHg) & $121.6 \pm 18.5$ & $126.8 \pm 18.3$ & $122.9 \pm 17.2$ & $125 \pm 19.7$ & 0.002 \\
\hline DBP (mmHg) & $75.1 \pm 10.2$ & $77.1 \pm 10.7$ & $77 \pm 10.2$ & $78.8 \pm 11.7$ & $<0.001$ \\
\hline eGFR $<60 \mathrm{~mL} / \mathrm{min}$ & $420(23.2 \%)$ & $44(28.6 \%)$ & $194(20.3 \%)$ & $17(23.3 \%)$ & 0.10 \\
\hline All-cause mortality & $338(18.5 \%)$ & $33(21.3 \%)$ & $163(17 \%)$ & $23(31.1 \%)$ & 0.030 \\
\hline $\begin{array}{l}\text { Coronary artery disease and } \\
\text { cerebral vascular accident }\end{array}$ & $127(7 \%)$ & $10(6.5 \%)$ & $69(7.2 \%)$ & $7(9.5 \%)$ & 0.86 \\
\hline
\end{tabular}

Abbreviations: BMI, body mass index; DBP, diastolic blood pressure; DM, diabetes mellitus; eGFR, estimated glomerular filtration rate; HR, heart rate; SBP, systolic blood pressure; SD, standard deviation 


\section{Table 4 (on next page)}

Hazard ratio and $95 \%$ confidence interval of the heart rate for adverse outcomes. 


\begin{tabular}{|c|c|c|c|c|}
\hline & \multicolumn{4}{|l|}{ Outcome } \\
\hline & All-cause mortality & $P$-value & $\begin{array}{l}\text { Coronary artery disease and } \\
\text { cerebral vascular accident }\end{array}$ & $P$-value \\
\hline Events (per 1000 person-years) & 14.36 & & 5.59 & \\
\hline \multicolumn{5}{|l|}{ Adjusted hazard ratio (95\% CI) } \\
\hline \multicolumn{5}{|l|}{ Model 1} \\
\hline \multicolumn{5}{|l|}{ Baseline HR (categorical) } \\
\hline (Group 2 vs. group 1) & $0.89(0.70-1.13)$ & 0.33 & $1.09(0.76-1.57)$ & 0.63 \\
\hline (Group 3 vs. group 1) & $0.95(0.75-1.19)$ & 0.63 & $0.88(0.60-1.28)$ & 0.49 \\
\hline (Group 4 vs. group 1) & $0.96(0.75-1.22)$ & 0.72 & $0.76(0.51-1.15)$ & 0.19 \\
\hline Baseline HR (per 5 beats per minute) & $0.99(0.96-1.04)$ & 0.85 & $0.95(0.89-1.01)$ & 0.11 \\
\hline Final HR (per 5 beats per minute) & $1.05(1.02-1.09)$ & 0.006 & $1.00(0.94-1.06)$ & 0.98 \\
\hline \multicolumn{5}{|l|}{ Final HR (categorical) } \\
\hline (Group 2 vs. group 1) & $1.06(0.84-1.34)$ & 0.61 & $0.86(0.59-1.25)$ & 0.43 \\
\hline (Group 3 vs. group 1) & $1.07(0.84-1.37)$ & 0.59 & $1.04(0.72-1.51)$ & 0.84 \\
\hline (Group 4 vs. group 1) & $1.28(1.02-1.60)$ & 0.035 & $0.96(0.66-1.39)$ & 0.81 \\
\hline$\Delta \mathrm{HR}$ (per 5 beats per minute) & $1.02(0.97-1.07)$ & 0.41 & $0.93(0.86-1.00)$ & 0.033 \\
\hline \multicolumn{5}{|l|}{ GBTM of HR } \\
\hline (Decreased vs. stable) & $1.06(0.74-1.52)$ & 0.75 & $0.90(0.47-1.72)$ & 0.75 \\
\hline (Mildly increased vs. stable) & $0.94(0.78-1.14)$ & 0.52 & $1.06(0.79-1.43)$ & 0.69 \\
\hline (Markedly increased vs. stable) & $1.86(1.22-2.84)$ & 0.004 & $1.54(0.72-3.30)$ & 0.27 \\
\hline $\mathrm{CV}$ of $\mathrm{HR}$ & $1.97(0.59-6.57)$ & 0.27 & $0.76(0.12-5.05)$ & 0.78 \\
\hline \multicolumn{5}{|l|}{ Model 2} \\
\hline \multicolumn{5}{|l|}{ Baseline HR (categorical) } \\
\hline (Group 2 vs. group 1) & $0.89(0.70-1.13)$ & 0.32 & $1.01(0.70-1.47)$ & 0.95 \\
\hline (Group 3 vs. group 1) & $0.90(0.71-1.14)$ & 0.38 & $0.82(0.55-1.20)$ & 0.30 \\
\hline
\end{tabular}




\begin{tabular}{|c|c|c|c|c|}
\hline (Group 4 vs. group 1) & $0.92(0.72-1.17)$ & 0.50 & $0.74(0.49-1.12)$ & 0.15 \\
\hline Baseline HR (per 5 beats per minute) & $0.99(0.95-1.03)$ & 0.65 & $0.95(0.89-1.01)$ & 0.11 \\
\hline Final HR (per 5 beats per minute) & $1.02(0.98-1.06)$ & 0.47 & $0.97(0.90-1.03)$ & 0.27 \\
\hline \multicolumn{5}{|l|}{ Final HR (categorical) } \\
\hline (Group 2 vs. group 1) & $1.17(0.91-1.51)$ & 0.22 & $0.94(0.62-1.42)$ & 0.77 \\
\hline (Group 3 vs. group 1) & $1.00(0.77-1.31)$ & 0.98 & $0.95(0.63-1.44)$ & 0.82 \\
\hline (Group 4 vs. group 1) & $1.09(0.85-1.41)$ & 0.51 & $0.81(0.54-1.21)$ & 0.30 \\
\hline$\Delta \mathrm{HR}$ (per 5 beats per minute) & $1.02(0.97-1.08)$ & 0.45 & $0.92(0.85-1.00)$ & 0.05 \\
\hline \multicolumn{5}{|l|}{ GBTM of HR } \\
\hline (Decreased vs. stable) & $1.02(0.71-1.46)$ & 0.94 & $0.81(0.42-1.55)$ & 0.52 \\
\hline (Mildly increased vs. stable) & $0.92(0.76-1.11)$ & 0.39 & $0.98(0.72-1.32)$ & 0.87 \\
\hline (Markedly increased vs. stable) & $1.80(1.18-2.76)$ & 0.007 & $1.45(0.67-3.12)$ & 0.34 \\
\hline $\mathrm{CV}$ of $\mathrm{HR}$ & $1.88(0.56-6.36)$ & 0.31 & $0.81(0.11-5.72)$ & 0.83 \\
\hline
\end{tabular}

Abbreviations: $\mathrm{CI}$, confidence interval; $\mathrm{HR}$, heart rate; GBTM, group-based trajectory model; $\mathrm{CV}$, coefficient of variance

Model 1: Adjusted for age, sex

Model 2: Adjusted for age, sex, systolic blood pressure, diastolic blood pressure, diabetes mellitus, smoking, alcohol, cholesterol, body mass index, estimated glomerular filtration rate $<60 \mathrm{~mL} / \mathrm{min} / 1.73 \mathrm{~m}^{2}$ 


\section{Table 5 (on next page)}

Hazard ratio and 95\% confidence interval of the heart rate for adverse outcomes according to the heart rate trajectories based on repeated measurements of three heart rates. 


\begin{tabular}{|c|c|c|}
\hline All-cause mortality & $P$-value & $\begin{array}{l}\text { Coronary artery disease and } P \text {-value } \\
\text { cerebral vascular accident }\end{array}$ \\
\hline
\end{tabular}

\section{Model 1}

\section{GBTM of HR}

Decreased vs. stable

$1.13(0.84-1.54)$

0.42

$0.90(0.52-1.57)$

0.71

Mildly increased vs. stable

$0.92(0.76-1.12)$

$0.41 \quad 1.13(0.84-1.51)$

0.42

Markedly increased vs. stable

$1.73(1.19-2.53)$

0.004

$1.16(0.54-2.48)$

0.71

\section{Model 2}

GBTM of HR

Decreased vs. stable

Mildly increased vs. stable

Markedly increased vs. stable
$1.09(0.80-1.48)$

$0.91(0.75-1.10)$

$1.62(1.10-2.38)$
$0.59 \quad 0.85(0.49-1.48)$

$0.34 \quad 1.00(0.74-1.35)$

$0.015 \quad 0.87(0.40-1.88)$
0.56

1.00

0.71

Abbreviations: CI, confidence interval; HR, heart rate; GBTM, group-based trajectory model; CV, coefficient of variance

Covariates: age, sex, systolic blood pressure, diastolic blood pressure, diabetes mellitus, smoking, alcohol, cholesterol, body mass index, estimated glomerular filtration rate $<60 \mathrm{~mL} / \mathrm{min} / 1.73 \mathrm{~m}^{2}$, and atrial fibrillation

Model 1: Adjusted for age, sex

Model 2: Adjusted for age, sex, systolic blood pressure, diastolic blood pressure, diabetes mellitus, smoking, alcohol, cholesterol, body mass index, estimated glomerular filtration rate $<60 \mathrm{~mL} / \mathrm{min} / 1.73 \mathrm{~m}^{2}$ 